\title{
Influence of Molecular Weight Average, Degree of Crystallinity, and Viscosity of Different Polyamide PA12 Powder Grades on the Microstructures of Laser Sintered Part
}

\author{
WAY Yusoff ${ }^{\mathrm{a}}$, Hanafi Ani ${ }^{\mathrm{b}}$, DT Pham ${ }^{\mathrm{c}}$, K. $^{\text {Dotchev }}{ }^{\mathrm{d}}$ \\ ${ }^{a, b}$ Kulliyyah of Engineering, International Islamic University Malaysia \\ ${ }^{c}$ School of Mechanical Engineering, University of Birmingham, United Kingdom \\ ${ }^{\mathrm{d}}$ School of Mechanical Engineering, University of Portsmouth, United Kingdom
}

\begin{abstract}
Laser Sintering (LS) allows functional parts to be produced in a wide range of powdered materials using a dedicated machine, and is thus gaining popularity within the field of rapid prototyping. It offers the user the ability to optimise part design in order to meet customer requirements with few manufacturing restrictions. A problem with LS is that sometimes the surface of the parts produced displays a texture similar to that of the skin of an orange (the socalled "orange peel" texture). The main aim of this research is to develop a methodology of controlling the input material properties of PA12 powder that will ensure consistent and good quality of the fabricated parts. Melt Flow Rate (MFR) and Gel permeation chromatography (GPC) were employed to measure the flow viscosity and molecular weight distributions of Polyamide PA12 powder grades. The experimental results proved that recycle PA12 powder with higher melt viscosity polymer has a higher entanglement with a longer molecule chain causes a higher resistance to flow which cause poor and rough surface finished on laser sintered part.
\end{abstract}

\section{Introduction}

In manufacturing, many products need to undergo increasing customisation, and a shortening of the manufacturing cycle time. This makes the time needed to produce prototypes one of the most important contributors to product development cycles. Because of the development and pre-production stages of rapid prototyping (RP), it is often necessary to have small batches of parts for testing and evaluation purposes [1]. Laser sintering (LS) is one of the most versatile Rapid Prototyping (RP) processes currently available.LS with Polyamide 12 (PA12) or Nylon 12 powders is a widely used technology for the rapid prototyping of functional parts and recently has become very attractive for the rapid manufacturing of small batches of plastic parts [2]. One of the main advantages of employing this technology is that the non-sintered powder can be recycled and reused for another fabrication. However, the fabricated part could be affected by rough and unacceptable surface texture. As a result, the parts may have to be scrapped and the build has to be repeated with a higher ratio of new material. Prior to this study,

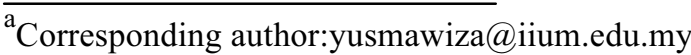

most previous research was carried out using PA12 as the focus for the study of the thermal, mechanical, and chemical properties which affect the part surface finish. One of the conclusions was that after approximately 7-8 builds the properties of the processed material were so badly deteriorated that it should be fully discarded and it is reported that the higher melt viscosity which causes higher warp, part shrinkage and slightly less part density [3-6]. However, the effect of the Molecular weight average and viscosity of different Polyamide PA12 powder grades in Laser Sintering process which influence the microstructure and surface finished of sintered part was not investigated.

\section{Fabrication of a part using laser sintering (LS)}

LS differs from conventional manufacturing processes in that it is an additive process which is able to produce a high degree of accuracy. In addition, no tooling, fixing, and reorientation are required during the process [6]. In this technology, an object is created layer by layer from heat-fusible solid fine powdered materials with heat generated from a $\mathrm{CO} 2$ laser. All of the PA12 powders are processed in a similar way during the LS process. As shown in figure 1, the PA12 particle size, 
from $10 \mu \mathrm{m}$ to $70 \mu \mathrm{m}$ and an irregular shape with a rough surface. They are exposed to long heating and cooling cycles, at temperatures very close to the material melting temperature in a Nitrogen rich atmosphere. At the end of the process, the temperature in the build is very high, from $140^{\circ} \mathrm{C}$ to $180^{\circ} \mathrm{C}$ [7]. These processing conditions cause physical and chemical changes of the powdered material and deterioration of its mechanical and thermal properties. The non-sintered powder can be recycled and reused for another fabrication after being blended with new material. If the amount of new powder is not sufficient or if only recycled material is used, then the fabricated parts experience variation in their quality, higher shrinkage, and a rough surface texture known as "orange peel" [8].

The objectives of this research are to improve the surface quality of parts produced by Laser Sintering (LS) and to reduce or eliminate the rough surface texture by investigating the Molecular weight average and viscosity of different Polyamide PA12 powder grades in Laser Sintering process.

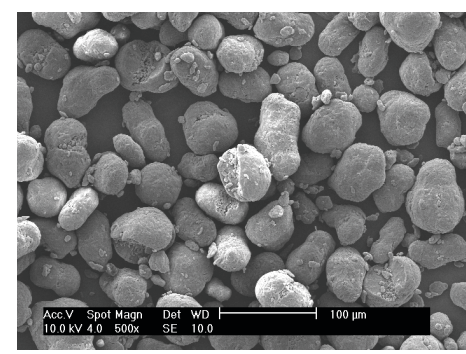

Figure 1. New PA12 powder

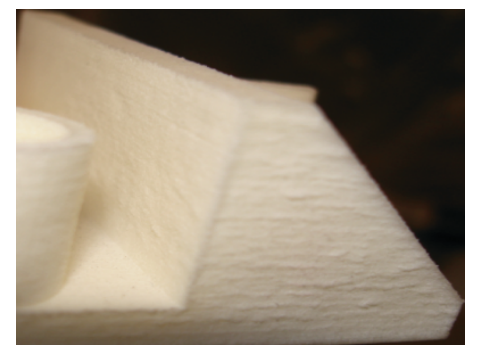

Figure 2. Rough surface texture

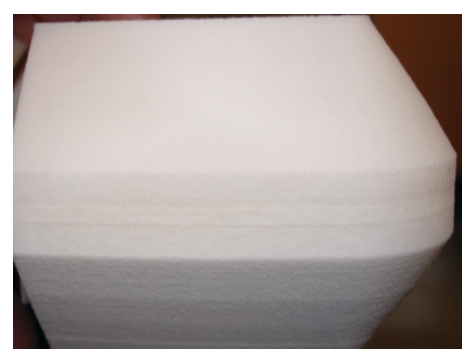

Figure 3. Good surface texture

As shown in figure 2 and 3, compare the poor surface texture and good part surface finished. This study focuses on one of the major limitation of LS. Current practice, which utilises recycled PA12, causes poor texture known as "orange peel" texture. Therefore, the quality of the powder used in the LS process is one of the most important parameter determining the final part quality [9].

\section{Research methodology}

The LS material investigated in this study is PA12based powder PA2200 supplied by EOS GmbH. The "New PA2200", 35\% new PA2200 mixed with 65\% once used recycled, once used recycled PA2200, twice recycled PA2200 and three times recycled PA2200 samples were prepared for testing. The basic polymer property which is measured by this test is the molten plastic flow at a particular shear stress (related to the applied load) and temperature according to ISO1133. In this case, the Melt Flow Rate (MFR) test provides a relatively fast and inexpensive method of measuring the rate of PA12 powder degradation because of the LS process.In this experiment, Gel permeation chromatography (GPC) is a primary technique for characterising polymers and measuring average molecular weight, It separates polymers on the basis of their size relative to the polymer molecules. The GPC experiments were performed according to ISO16014 standard. For all examinations, a thin layer of gold was sputtered on substrates using an auto sputter apparatus. Two pieces of equipment have been used consecutively. The first is a Bio-Rad SC500 for gold coating of the specimens and the second is an EMSCOPE SC500 for image capture. It is employed to characterise the individual powders and to analyse the surface morphology and microstructure of the sintered part.

\section{Results and analysis}

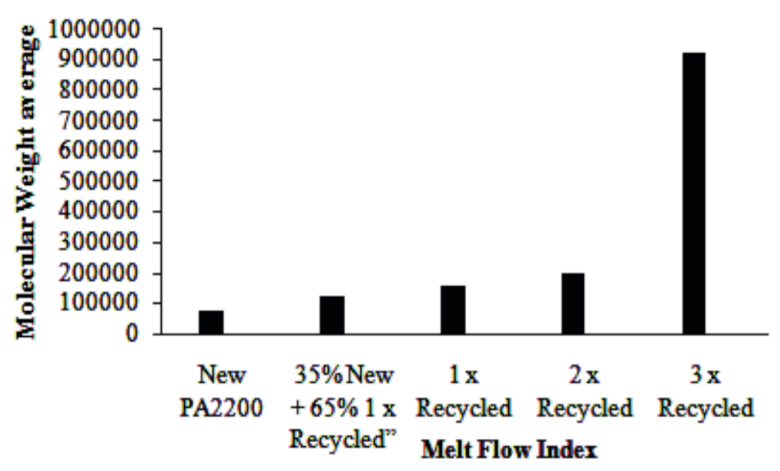

Figure 4. Melt flow index and Molecular weight average 


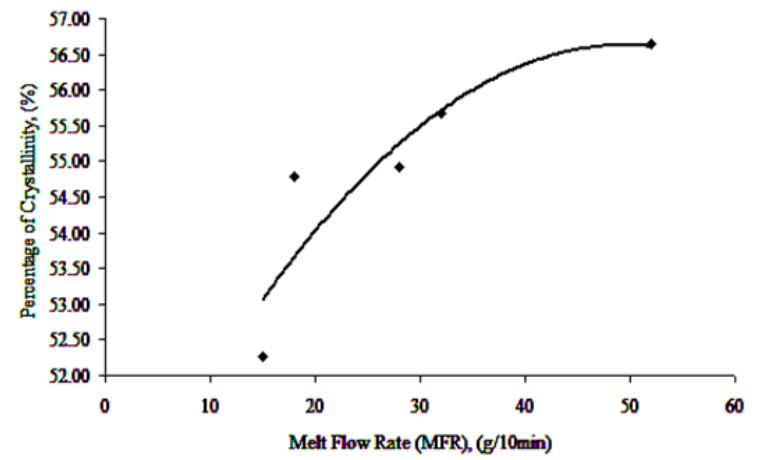

Figure 5. Melt flow index and crystallinity

As shown in figure 4 and figure 5 the relationship between the MFR and the percentage of crystallinity. The samples of "New PA2200" have larger degree of crystallinity and higher MFR compared to the samples of the older or recycled powder. The higher the temperature and longer time at which the powder is exposed to heat, the higher the chance that the molecule chains will become larger. This means that the longer the unsintered PA12 powder stays in the LS machine build cylinder during the build and cooling-down stages, the more the molecular chains would become larger. Ultimately, this causes an increase of the molecular weight and, therefore, an increase of the melt viscosity. It has been also found that there is a correlation between Molecular weight average and the degree of crystallinity of the PA12 powder, the longer the molecular chain, the higher the molecular weight where the higher Molecular weight average causes higher melt viscosity due to more efficient packing of the PA12 polymer chains which also leads to an increase in the LS part's shrinkage.

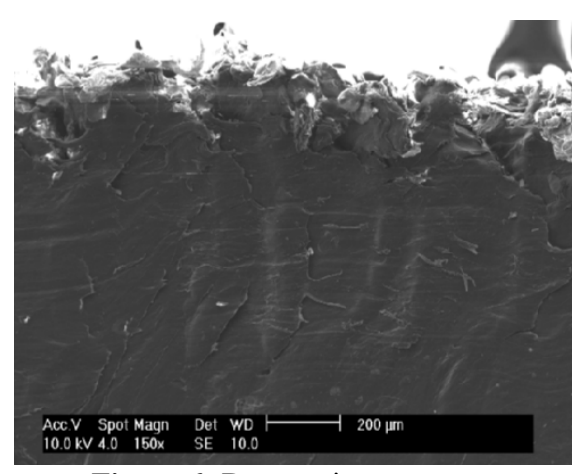

Figure 6. Dense microstructure

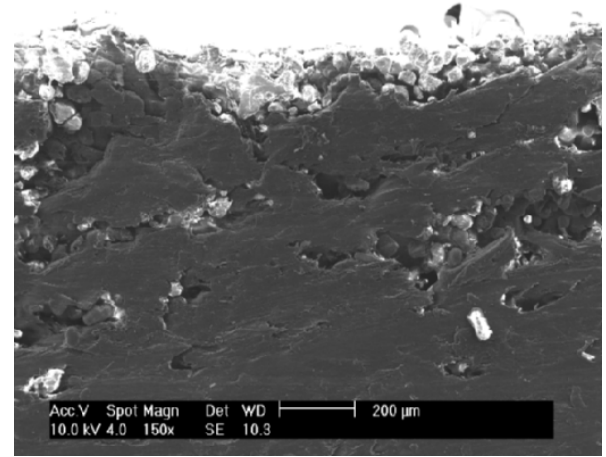

Figure 7. Inhomogeneous structure

The microstructures of laser sintered parts on the $\mathrm{x}$ y plane are as shown in Figure 6 and 7 . This result suggests that the use of refreshed powder results in the formation of microstructures with a highly dense morphology. In contrast, the cross sectional of an "orange peel" texture exhibits a very inhomogeneous structure after laser sintering. It is observed that cavities have different shapes and sizes, the distance between voids when employing twice-used powder is as little as $100 \mu \mathrm{m}$ to $160 \mu \mathrm{m}$ and it may clearly be seen that the average cavity size is approximately $100 \mu \mathrm{m}$ to $200 \mu \mathrm{m}$. This result proves that the higher quality MFR powder has shorter molecular chains. This causes it more easily to become liquid and to flow easily during the sintering process. The better PA2200 powder quality grades, the lower the Molecular average weight value.

\section{Conclusions}

The PA12 powder properties deteriorate due to the high temperature close to the material melting temperature for a long period of time through the LS building and cooling cycles. The melt viscosities of the polymer are found to be closely related to the molecular weight average and degree of crystallinity which affects the LS microstructure and part surface finished.

\section{Acknowledgement}

This research was fully funded by the EPSRC United Kingdom: GR/S75505/01 and collaboration with Manufacturing Engineering Centre (MEC), Cardiff University, Wales.

\section{References}

1. Shwe Pyi Soe, Daniel Roy Eyers, Wan Ahmad Yusmawiza, and Chi Hieu Le. Laser Sintering for the Fabrication of Architectural Models, Advances in Manufacturing and Materials Engineering: Advanced Materials Research, Vol.576 (2012),pp.637-640, ISSN: 1660-9336.

2. Pham D T, and Dimov S S, (2003) Rapid Prototyping and rapid tooling - the key enablers for rapid manufacturing, Proceedings of the Institution of Mechanical Engineers, Part B: 
Journal of Engineering Manufacture, Vol. 217, No 1, pp 1-24.

3. G.V. Salmoria , J.L. Leite, L.F. Vieira, A.T.N. Pires,C.R.M. Roesler (2012)Mechanical properties of PA6/PA12 blend specimens prepared by selective laser sintering, Polymer Testing Volume 31, Issue 3, Pages 411-416

4. Gornet T J (2002) Characterisation of Selective Laser sintering TM to Determine Process Stability, Proceedings of Solid Freeform Fabrication, Austin, Texas, pp 546-553.

5. T. Jollivet, A. Darfeuille, B. Verquin, S. Pillot (2009) Rapid manufacturing of polymer parts by selective laser sintering, International Journal of Material Forming, Springer, pp 697-700.

6. J.P. Kruth, G. Levy,F. Klocke, T.H.C. Childs (2007),Consolidation phenomena in laser and powder-bed based layered manufacturing, CIRP Annals - Manufacturing Technology,Volume 56, Issue 2,Elsevier, pp730-759.

7. Dimov S S, Pham D T, Lancan F and Dotchev K.D (2001) Rapid tooling applications of the laser sintering process, Rapid Prototyping Journal, Vol.4, No.4, pp 296-302.

8. D.T.Pham, K.D.Dotchev, WAY Wan Yusoff (2007), Improvement of part surface finishing in laser sintering by experimental design optimisation (DOE), 3rd Intelligent Production Machines and Systems Virtual International Conference, Elsevier,. ISBN: 9781904445524.

9. Wan Yusoff, A.J Thomas (2008) The Effect of a Laser Scanning Strategy and Energy Density on "Orange Peel" Phenomenon of Selective Laser Sintering Process (SLS), Proceedings of the 17th International Conference on Management of Technology, Dubai, UAE. 